\title{
HYPER-MSPACE; MULTIDIMENSIONAL EVOLUTIONARY-AGENTS MODELING AND ANALYSIS
}

\author{
Mohamed Dbouk ${ }^{1}$, Ihab Sbeity ${ }^{1}$, Hamid Mcheick $^{2}$, Haytham Douaihy $^{3}$ \\ ${ }^{1}$ Lebanese University, Faculty of Sciences (I), Beirut-Lebanon \\ mdbouk@ul.edu.1b, isbeity@ul.edu.lb \\ ${ }^{2}$ Université du Québec à Chicoutimi, Québec, Canada \\ Hamid Mcheick@uqac.ca \\ ${ }^{3}$ Lebanese University-DSST, Beirut-Lebanon, Master2R-Student \\ haythamdouaihy@gmail.com
}

\begin{abstract}
This paper stands for an analytical approach modelling and exploring an evolutionary-space oriented business-domains. The space that we consider is intended to be complex emerging a set of heterogeneous evolutionary business-agents (e.g. Factories, Restaurants, etc.); it accordingly stimulates an analytical multi-layered structure headed for a hyper-space navigational framework. Agents have multiple-states based on analytical characteristics (analytical dimensions), they are multidimensional and interdependable; they evolve, interact and transit. This approach aims to provide key features that illustrate the hyper-space framework basis and foundations. Finally, an instructive case study is given at the end of this document.
\end{abstract}

\section{KEYWORDS}

Evolutionary agents, Hyper-space modelling, Multi-State modelling, GIS-like frameworks, Business intelligence.

\section{INTRODUCTION}

Day by day, reactive and dynamic data are strongly solicited. This data may incorporate and stimulate gigantic amount of knowledge. The advance in technology, multidimensional and business intelligence analysis are extremely present in similar context.

However, things evolve in the nature, everything evolve and change states and conditions, e.g. an employee may become manager, president, etc., modern cities, construction sites, volcanic sites, etc., may have continuous stories and histories (images, video, maps, etc.); they evolve continually against time, urbanisations and civilizations.

Concepts of evolutionary space may, indeed, reside in lots of real-world events and business activities; environmental risk related issues such as flooding, forest (trees, etc.) evolutions, agricultural practices, are typical cases.

In our early MSpace platform [1], all considered agents are belong the same type, i.e. employers, buildings, etc. Moreover, the space itself is reduced to one and only one business context/environment (layer).

The ultimate goal of this work is to increase and enrich our MSpace platform with new hyperspace characteristics and functionalities; the space is multi-layered now and become so complex 
by emerging heterogeneous cooperative and evolutionary agents. However, dealing with such unconventional space architecture/structure is hard to undertake and reveal great challenges. The multi-layering concept leads to provide an intelligent and analytical vertical navigation.

The duty is to ultimately devise, on one hand, a strong and firm theory correlating the intended hierarchical structure; towards an analytical-oriented specialization/generalization strategy that incorporates the vertical navigational and analytical transitions. The duty, on the other hand, is to correlate interactions and behaviours of the considered heterogeneous agents each other, and also to adjust the inter/intra-events which trigger transitional evolutions.

The rest of the paper is organized as follow: in Section 2 we review background material, the early MSpace as well as problem statement are outlined and an overview of related works is also given in this section. In section 3, MSpace extension foundations and basis are deeply considered. Also, a deep modelling theory and navigation as well as visual solution are presented in this section. An experimental case study is also given in section 4. Finally, the outlines of future directions and conclusion are drawn in section 5.

\section{BACKGROUND AND PROBLEM STATEMENT}

\subsection{MSpace, an overview}

The early MSpace [1] (started with [2]) consists of an integrated tool tending to model and explore (tracking and monitoring) an evolutive and mutable space, it deals and emerges multidimensional and unconventional data, it mainly considers a collection of mutable entities (became agents), which are referenced by a structural features (dimensions), and tracked according to behavioural features (transitions, business rules).

Entities/Agents in MSpace are expected to move, transit, navigate, interact, etc. agents' behaviours reflect and/or materialize external events. Rather than temporal and location-based characteristics, they evolve in well organized behaviours. MSpace deals with contextual patterns (business profiles, figure 1), in which dimensions are pre-combined.

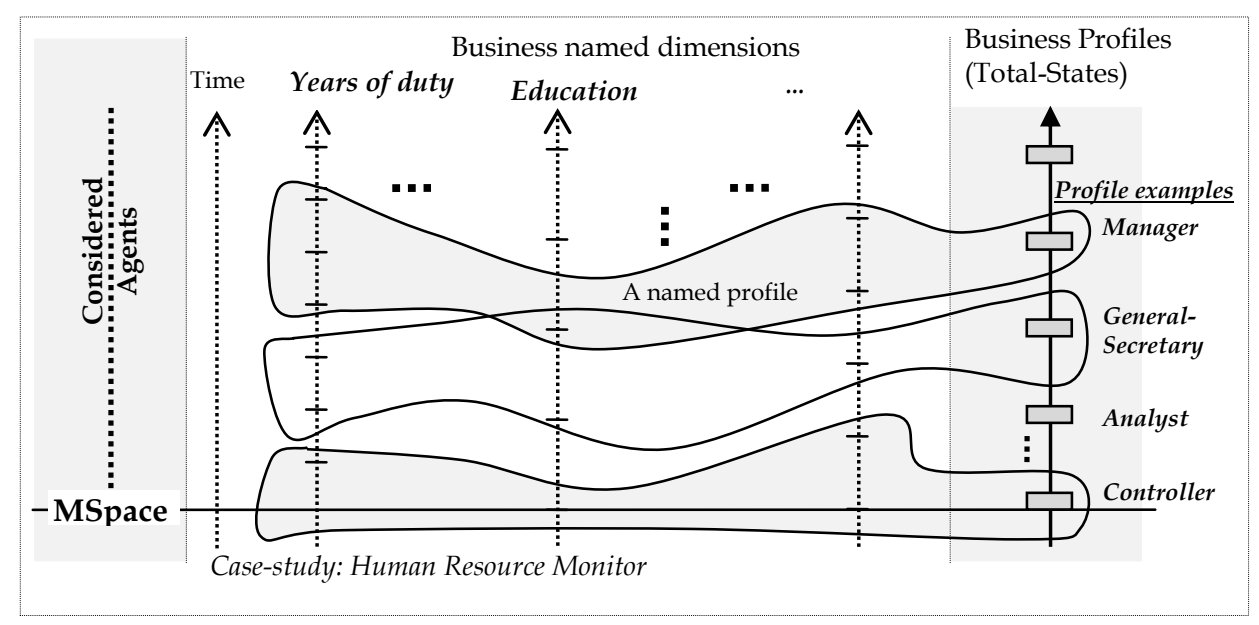

Figure 1. MSpace [1] platform basis and foundations

The ultimate goal, of "MSpace", is to enable entity (individual space's element) evolution tracking and monitoring. The duty is to predict, direct and ultimately correlate entities' behaviours and activities. 


\subsection{Problem position and related works}

In the practice, business-spaces are usually complex and considered entities/agents could reference different categories and classes, they may interact and influence each other, etc.

Consider the typical case of a modern city evolution analysis. To analyze and track the evolution of the city, we have, indeed, to record and analyze the urbanization activities from multiple perspectives; i.e. the existence of a Factory may infect healthy conditions, the existence of buildings requires the presence of streets, the existence of 20 buildings or more requires the presence of gardens, etc.

Here the space may contain heterogeneous and inter-dependable agents; it is complex and hard to undertake. The concept of subdividing the space into bordering areas/subspaces and or applying the multi-layering approach may be helpful, but dealing with such cases requires deep modelling efforts. The idea, indeed, behind this case is to exhibit the big challenges that we are facing where the space is qualified as complex.

However, in the early above approach [1], complex cases are not yet solved. Rather than the elegant and hopeful outcomes, the experiments show that we need to deploy more efforts in order to enlarge the analytical spectre of this approach.

Obviously, dealing with evolutionary-objects or agents is sometimes synonymous to deals with moving objects or agents on one hand and to consider the spatiotemporal dimension/concept on the other hand.

- $\quad$ The FuMMO model proposed by [5] works indeed with geo-features, the moving objects data model "Balloon" [6] deals with spatiotemporal changes; it provides an integrated support for both historical and future movements of moving objects. In contrast to basic "FuMMO" model, "Balloon" states for emerging both historical and future moving-objects" movements.

- "Hermes" [4] deals with dynamic objects that change locations, shape and size, either discretely or continuously over time.

- In the land use model presented in [3], the authors use discrete irregular objects as an autonomous spatial entity. They use the basic elements of Cellular Automata: space, neighbourhood, and transition rules, state, and time, and demonstrate that the proposal can be considered as a new paradigm for urban simulation. Here, the world is considered as a series of entities located in space. An autonomous active object or agent is a digital representation of all part of an entity. In this model, the transition rules specify the behaviour of cells between time-step evolutions, deciding the future conditions of cells based on a set of fixed rules. However, the state of the object changes is based on the adaptation of an entity to its environment. Also the time variant is considered as an element in this approach.

Briefly, we plan, in the next sections, to extend the early MSpace approach by adding the so called hyper-features, and functionalities. 


\section{HYPER-MSPACE}

\subsection{Complex space modelling}

The new-extended initiative "Hyper-MSpace" emerges indeed features and concepts which are considered by the above premature approaches ([3], [4], [5] and [6]), it inherits concepts from multi-agents systems, but remaining different on the way to define agents.

Here, we are going to devise a deep theory leading to model that we call "Hyper-MSpace"; rules that govern agents interactions, space subdivision rules, multi-layering space constitution, vertical navigational rules (summarising, zooming, etc.), etc. As a result, we state here for a complete complex-space building and exploring process; we consider answering questions like: "how the space should be partitioned thematically", "how entities could interact and influence each other", "how could navigate through different levels", etc.

Basic assumptions: Hyper-MSpace (HMSpace) consists of an integrated space, it emerges several agent categories, consequently it incorporates a set of MSpaces (figure 2)

Formally, HMSpace $(\mathrm{C} 1, \mathrm{C} 2, \ldots, \mathrm{Ci}, \ldots \mathrm{Cn})=<\mathrm{MS1}, \mathrm{MS} 2, \ldots, \mathrm{MSi}, \ldots, \mathrm{MSn}>$

- $\mathrm{MS}_{\mathrm{i}}=\operatorname{MS}\left(\mathrm{C}_{\mathrm{i}}\right) ; \quad$ MSpace (figure 1) is incorporating entities of type $\mathrm{C}_{\mathrm{i}}$. Consequently, it should instantiate all mspace pre-elaborated features.

- $\mathrm{Ci}$ is an agent multidimensional type

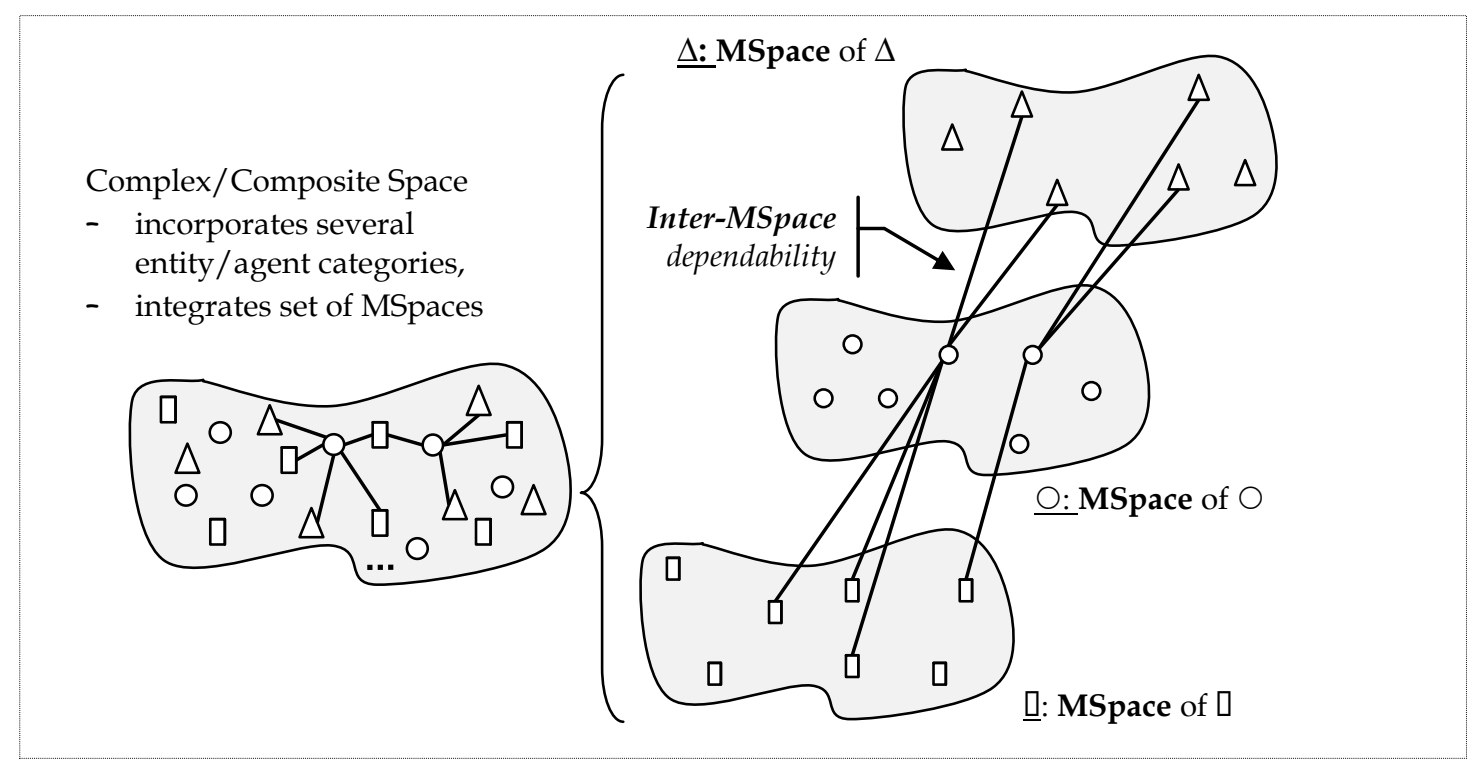

Figure 2. HMSpace basic constitutions

\section{Inter-dependability modelling:}

Emerged agents in HMSpace are intended to influence each other. An evolutionary agent (also called receiver) may depend on other agent/s (called master). Accordingly, an oriented mutual association is so established between master-agents and receiver-agents. Consequently, a huge inter-agents dependability network could be established, it represents a derivation /instantiation 
of an extrapolated network called "inter-agents dependability diagram (IADD)" (figure 3). An IADD consists of an oriented graph; nodes represent the involved mspaces and the arrows represent the inter-dependence relationships between agent-instances.

However, when a master-agent " $\square$ " or " $\Delta$ " evolve, it could affect (influence) all dependent receiver-agents " $\bigcirc$ " (figure 4). Based on the mspace firm foundations, the receiver-agent evolve throughout dimensions, consequently:

- A master-agent affects receiver-agents on one of their pre-defined dimensions. Arrows in dependability-diagram should be labeled with the given receiver-agent dimension.

- Master-agent may affect receiver-agents on many dimensions.

- Master and receiver agents may share (be belong) same mspace, towards reflexive dependability.

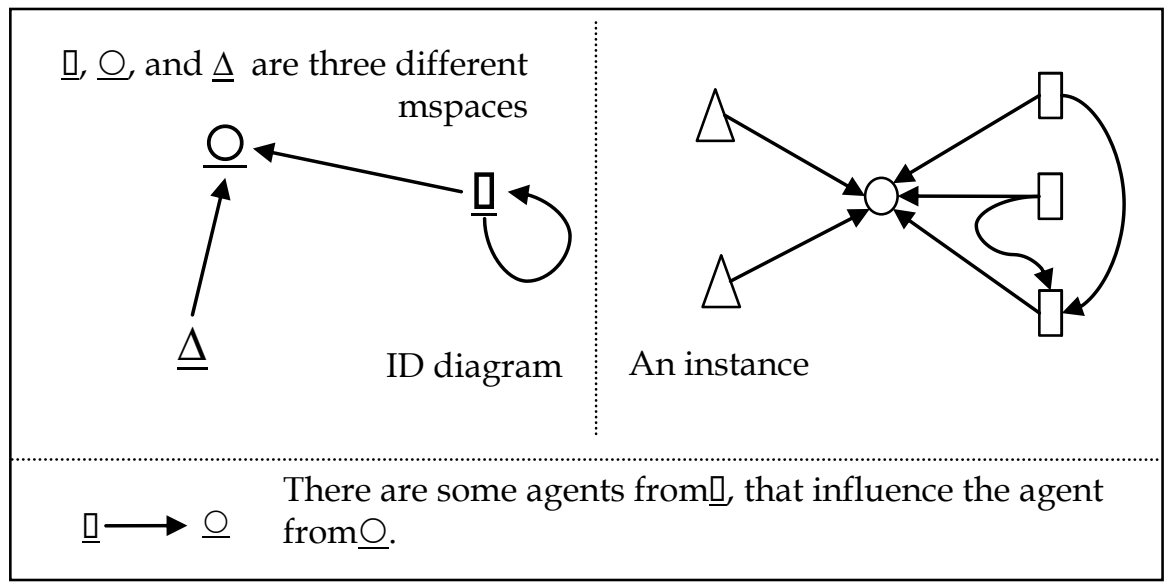

Figure 3. Inter-mspaces dependencies diagram

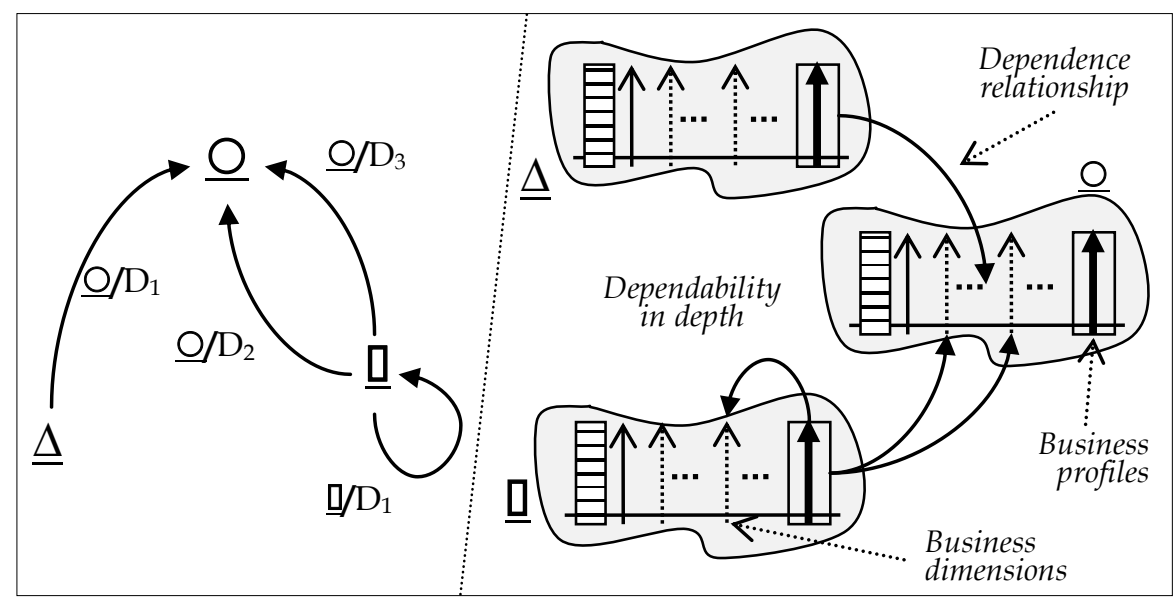

Figure 4.a. HMSpace platform basis and foundations

\section{Materializing inter-dependability:}

The association that links master-agents and receiver-agents can be materialized using the Observer/Observable design pattern; it is event-driven based. 
Here, receiver-agents play the role of observers, and variations or evolutions on one master-agent are triggered. Consequently, local-transitions from state to state of a receiver-agent (on the given dimension like D3, figure 4) are now directly linked to the master agent's total-transitions (locally tracked in the given mspace; business-profiles).

\section{Formally;}

$\Delta$ is a master-agent / observable pre-declared in mspace $\underline{\Delta}$ (e.g. $\underline{\Delta}: \Delta)$

$\bigcirc$ is a receiver-agent / observer pre-declared in mspace $\underline{\bigcirc}$ (e.g. 으: $\bigcirc)$

\section{Protocol:}

1. $\Delta$ Transits form local-state to a new state, consequently, a new total-state should be generated in $\underline{\Delta}$.

2. The new $\Delta$ state is observed by $\bigcirc$ and triggered.

3. $\bigcirc$ applies a dedicated transition-function "O.Dim $\tau$ ", it analyses the $\Delta$ new state and decides to transit from its old state to another local state on the given dimension, it should generate a new total-state in $\underline{\bigcirc}$, and then new business-profile may be generated as well.

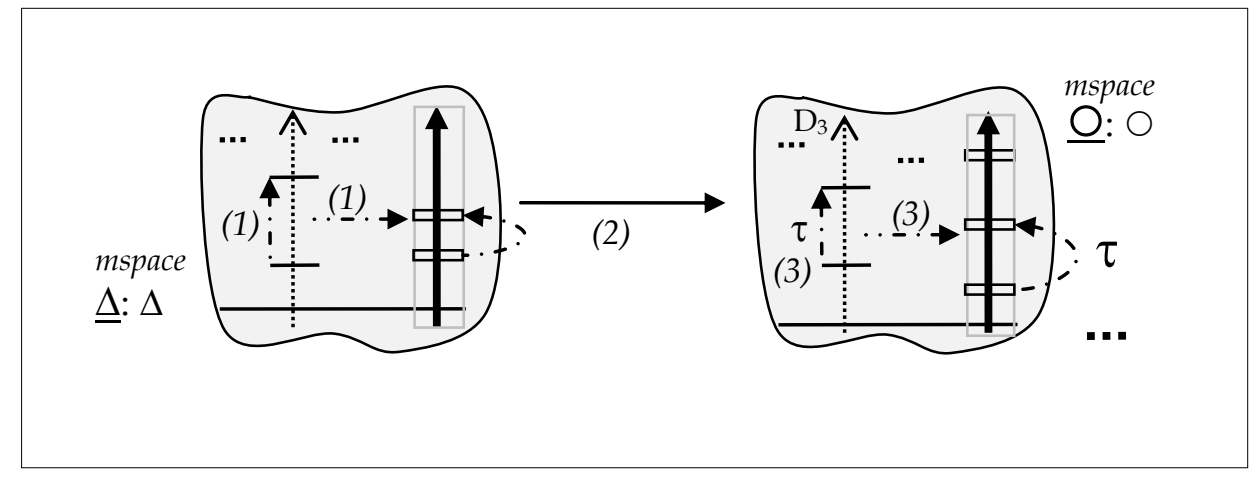

Figure 4.b. HMSpace platform basis and foundations

\section{Transition-function formulation and generalization:}

O.D3 $\tau(\Delta)$; transition of agent $\bigcirc$, on dimension "D3" taking into account the $\Delta$ evolutions. More general:

$-\bigcirc$ may be concerned with more than one $\Delta ; \bigcirc . \mathbf{D 3} \tau\left(\Delta_{1}, \Delta_{2}, \ldots, \Delta_{\mathbf{i}}, \ldots, \Delta_{\mathbf{n}}\right)$

$-\bigcirc$ may be linked to more than one agent-mspaces; here and aggregation " $\Sigma$ " must be applied: $O . \Sigma(\operatorname{Di} \tau(\ldots))$.

However, those dependability functions are domain-dependant and should be written by the domain analytical experts. As a result, the HMSpace platform has become open.

\section{Dynamic aspect:}

According to the pre-elaborated IADD (figure 3), consider that agent $\bigcirc_{4}$ depends on $\left\{\left\langle\Delta_{l}, \Delta_{3}\right.\right.$, $\left.\left.\Delta_{5}\right\rangle,\left\langle\mathrm{Q}_{1}, \mathrm{Q}_{4}, \mathrm{Q}_{6}\right\rangle\right\}$, and the new agent $\Delta_{2}$ depends, in its turn, on $\mathrm{O}_{4}$, the following scenarios should be considered:

- On cascade-evolution; if one of the $\left\{\left\langle\Delta_{1}, \Delta_{3}, \Delta_{5}\right\rangle,\left\langle\mathrm{Q}_{1}, \mathrm{Q}_{4}, \mathrm{Q}_{6}\right\rangle\right\}$ evolves, the $\mathrm{O}_{4}$ should be 
affected and may change state. Consequently, $\triangle_{2}$ will (by transitivity) evolve as well, and so on. All related agents will evolve, toward an on cascade-evolution.

- Dependability-pertinence: agents evolve continually, after each evolution-round, the IADD instances should be re-examined and evaluated. As a result, $\mathrm{O}_{4}$ for example may lose (become free of) some links, and also new links may re-appear with respect to the pre-elaborated IADD, i.e. $\bigcirc_{4} \leftarrow\left\{\left\langle\Delta_{l}, \Delta_{5}\right\rangle,\left\langle\square_{1}, \square_{5}, \square_{6}\right\rangle\right\}$.

\section{Visual and navigation issues}

The early mspace platform [1] consists of a set of pre-elaborated visual and analytical operations. Here in the HMSpace context and based on the given IADD, each mspace is represented as an analytical-node, and the inter-dependability links represent the firm foundation of two additional operations:

- Navigation throughout mspaces: allowing end-users to explore the whole considered space (hmspace), and to discover mspaces one by one, this is to materialize relationships "master-receiver" influences, e.g. $\Delta_{1} \rightarrow \mathrm{O}_{4}$

- Analytical zooming: this is to explore a given agent or mspace and going forward on a specific dimension. Here, the end-user must be able to discover and learn about the transition-modalities.

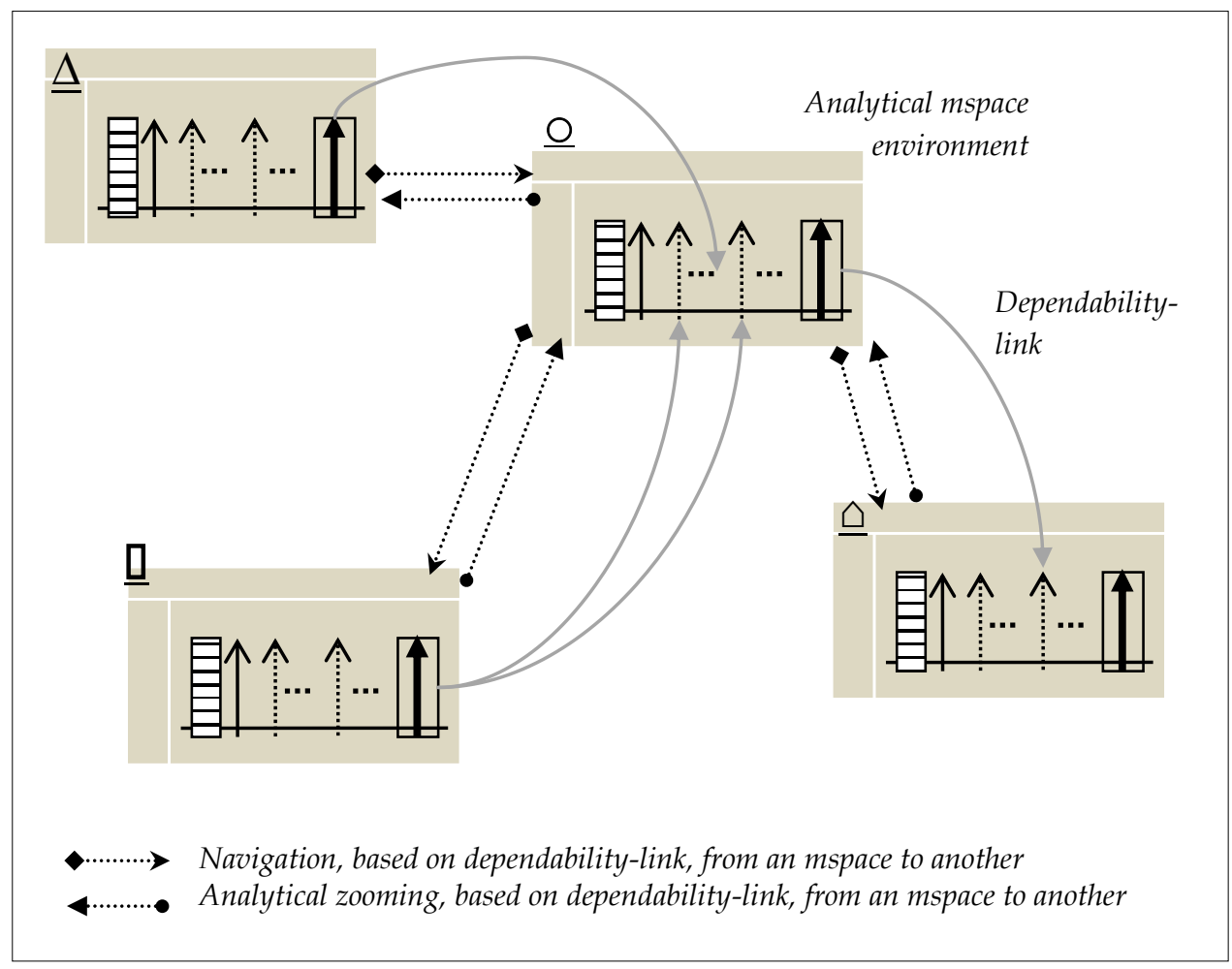

Figure 5. HMSpace end-user display basis and foundations

As a result, the above operations are complementary to each other. The IADD is, continually, considered as the main display that summarizes hyper-space new essential operations. 


\subsection{Hyper-Space building process}

The building process consists of the following:

a. Identify and consider the involved agent-mspaces and all descriptive and analytical features or dimensions,

b. analyze and find-out the inter-dependability relationships that relate the above mspaces,

c. Put mspaces and relationships together in a unique IADD,

d. re-adjust the above mspaces according to the founded relationships,

e. designate labels that governing the above dependability relationships,

f. Draw up (pseudo code) the transition-functions one by one.

g. Implementation phase: put the given IADD into operational platform.

h. Apply analytical and predictable scenarios.

Practically, the process is software-engineering-like, it requires key persons to be applied, and they should emerge technical-skills as well as deep knowledge in the considered businessdomain.

\section{EXPERIMENTAL CASE STUDY AND OBSERVATION}

Consider the case of patients tracking and monitoring. Here, the main challenge is to produce and implement the equivalent IADD.

However, we are going to focus and deal with patients (figure 6) and their analytical dimensions; administered-medicines and properties, treatment modalities, body building issues, climate issues, residency (home-residence), etc.

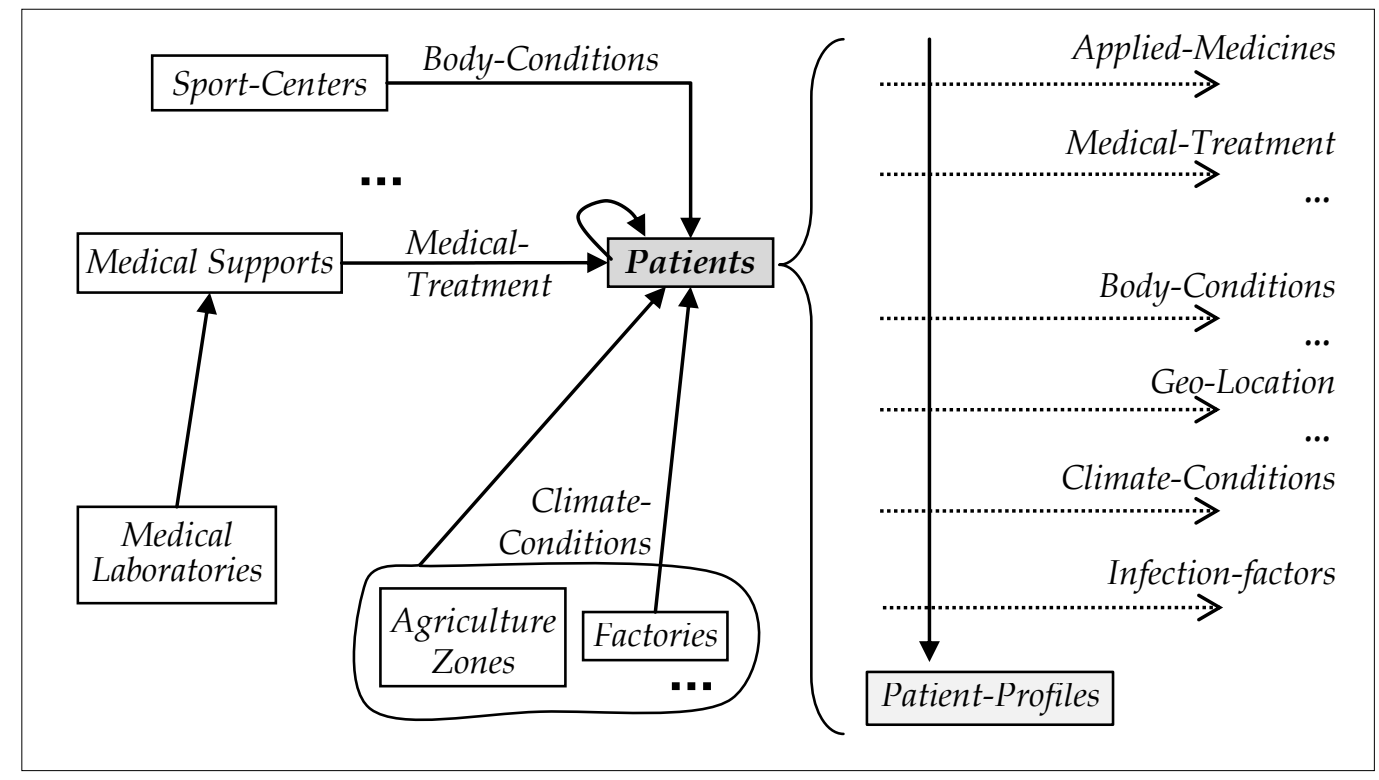

Figure 6. Patients tracking case study 
If, for example, patients are concerned with the climate-variations and pollution degrees, then we have to find out the concrete agents that influencing the climate-conditions like "AgricultureZones" and "Factories" (figure 6). The same conceptual analysis leads to consider "MedicalSupports" and "Medical-Laboratories", "Sport-Centers", etc., each of which relates (labeled with) one analytical dimension.

To conclude, first conceptual experiments show that, extra-agents could be grouped/aggregated into some abstract-agent-types, this is because those extra-agents share (influence) same analytical-dimensions, e.g. "Climate-agent" may aggregate "Agriculture-Zones" and "Factories". Finally, the experiments also show that we are pressed to deploy additional efforts that lead to implement the above theory and concretely validate the delivered process.

\section{CONCLUSION}

The early "MSpace" approach that we proposed in [1] provides a flexible modeling and analytical framework, but it deals and considers only one agents type. However, extending this approach represented, for us, the big challenge.

We stated, during the above work, for a complex-space called HMSpace (Hyper MSpace) that emerges heterogeneous agent-types and deals with inter-agents interactions. As a result, we divested a deep inter-agents dependability theory which was formulated with a new elegant UML-like structure called IADD (Inter-Agents Dependability Diagram).

Rather than the hopeful outcomes, the experiments show that we need to deploy more efforts at the validation phase. Finally, extending the early MSpace software-prototype outlines our future research.

\section{ACKNOWLEDGEMENTS}

The authors gratefully acknowledge support from the Lebanese University Research administration, Doctoral School of Sciences \& Technologies (EDST) - Beirut - Lebanon

\section{REFERENCES}

[1] Dbouk, M., Mcheick, M. \& Sbeity,I. (2012) "MSpace: An Emergent Approach, Modelling and Exploring Mutable Business-Entities", Advances and Applications in Mobile computing, Publisher InTech, Edited by Adem Karahoca, Chapter 6, pp 115-136.

[2] Dbouk, M. \& Sbeity, I. (2009) "GeoSEMA: A Modelling Platform, Emerging GeoSpatial-based Evolutionary and Mobile Agents. World Congress on Science, Engineering and Technology (WCSET 2009); Conf. Proceedings ISSN: 2070-3740, March 23-25, 2009, Hong Kong, China.

[3] Hammam, Y., Moore A., Whigham P. \& Freeman C., (2004) "Irregular vector-agent based simulation for land-use modelling". In 16th Annual Colloquium of the Spatial Information Research Centre (SIRC 2004: A Spatio-temporal Workshop), 29-30 November 2004, Dunedin, New Zealand, pp. 103-116.

[4] N. Pelekis, Y. Theodoridis, S. Vosinakis, T. Panayiotopoulos, "Hermes - A Framework for LocationBased Data Management", EDBT 2006, Springer Lecture Notes in Computer Science, Vol. 3896, pp.1130-1134.

[5] Praing, R. \& Schneider M. (2007). A Universal Abstract Model for Future Movements of Moving Objects. In AGILE International Conference on Geographical Information Systems, 2007. 
[6] Praing, R. \& Schneider M. (2007). Modeling historical and future movements of Spatio-temporal objects in moving objects databases. In proceedings of the 16th ACM Conf. on Information and Knowledge Management (CIKM), p183-192, Lisbon- Portugal, Nov. 6-10, 2007.

\section{AuTHORS}

Mohamed Dbouk, the main author, is a full time Professor at the Lebanese University (Beirut-Lebanon), he coordinates a master-2 research degree "IDSS: Information and Decision Support Systems". He received his PhD from "Paris-Sud", France, 1997. His research interests include software engineering and information systems related issues, geographic information systems, data-warehousing and data-mining.

Ihab Sbeity is a full time Associative Professor at the Lebanese University - Faculty of Sciences I -Department of Computer Science. He is member of the IDSS teaching-team. He received a $\mathrm{PhD}$ from the "Institut National Polytechnique de Grenoble", France. His $\mathrm{PhD}$ works are related to Performance Evaluation and System Design. His research interests include software performance engineering, UML modeling, multi-criteria decision making and information systems.

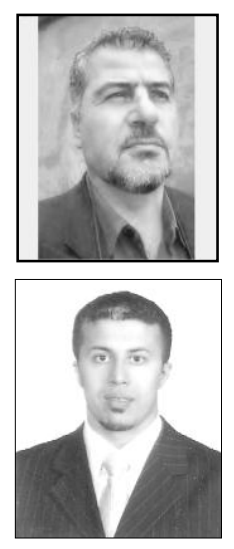

Hamid Mcheick is currently full time Associate Professor in Computer Science Department at the University of Quebec at Chicoutimi (UQAC), Canada. He received a Master's degree and PhD in Software Engineering and Distributed System from Montreal University, Canada. He is interested in software development and architecture for enterprise applications as well as in separation of concerns (component, services, aspect, etc.). His research is supported by many research grants he has received from the Canadian Government, University of Montreal, "Centre de Recherche informatique de Montreal (CRIM)", University of UQAM, and University of UQAC.

Haytham Douaihy attended the Graduate School of Sciences \& Technology in the Lebanese University, for earning a Master degree Research in Information Systems. In October 2007, He started working at "Path-Solutions", a Leading Software Provider company in the Banking Industry, In June 2011; he got a position as a Senior Web Developer at a Software Provider company in the Banking Industry: 'Xperteam'.
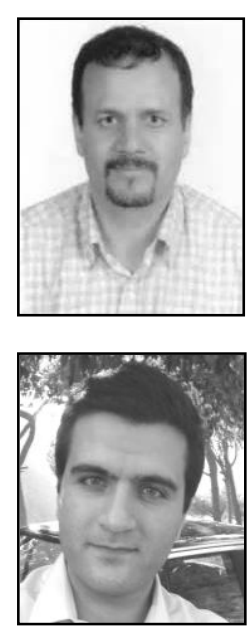